in the community and working together to design and deliver solutions

\section{METHODS/STUDY POPULATION:}

- Identify measures in the 3 domains of vulnerability, health and assets for the targeted neighborhoods and conduct bivariate descriptive statistics and multivariable regression analyses to investigate association between measures of vulnerability and health outcomes.

- Initiate relationships with leaders and residents in targeted neighborhoods

- Locate organizations working in targeted neighborhoods through online mapping software and word-of-mouth at neighborhood events, and created a spreadsheet with contact information.

- Conduct multidisciplinary assessment (i.e. key informant interviews, focus groups, town hall meetings) of the targeted neighborhood.

- Iteratively synthesize assessments to develop areas of interest and relevance to the community.

- Develop a road map for solutions identified by the community.

RESULTS/ANTICIPATED RESULTS: The results from the environmental scan conducted will be displayed in a report and visual "map" of health outcomes and health determinants, including assets and barriers for the targeted neighborhoods. The research team will use results from the environmental scan coupled with listening activities including attendance at community events, key informant interviews and focus groups to develop relationships and strong collaborations with the targeted neighborhood stakeholders. The relationship building between the research team and community will provide increased trust and engagement that will further enhance the effectiveness of the assessments completed with the targeted neighborhood. The assessments will help to empower communities to develop sustainable solutions and drive future work that will lead to future grant applications and largerscale implementation in other community impact hub neighborhoods. DISCUSSION/SIGNIFICANCE OF IMPACT: Through the community impact hub work, we will develop collaborative efforts with targeted neighborhoods with the greatest health inequities in the Marion County area. In partnership with these neighborhoods, we will build a foundation - a network of community collaborators and a focused plan - upon which we will improve the health outcomes of residents while learning best practices on how to do so.

4255

Indiana Clinical and Translational Sciences Institute (CTSI) - CTSA-wide podcast opportunity

Christine Drury ${ }^{1}$, and Aaron E. Carroll, MD ${ }^{1}$

${ }^{1}$ Indiana University School of Medicine

\section{OBJECTIVES/GOALS:}

- The podcasts highlight work from our partners: Indiana University, Purdue University and the University of Notre Dame.

- Our goal is to expand our podcast reach to include work from at least three additional CTSAs, as well as highlighting the benefits of translational research to the public.

\section{METHODS/STUDY POPULATION:}

- Aaron E. Carroll, is the director of Education and Workforce Development for the Indiana CTSI and a popular writer covering health, research, and policy for The New York Times. He is host of the Indiana CTSI-sponsored Healthcare Triage podcasts as well as the Healthcare Triage YouTube show, with 340,000 subscribers. We will leverage his audience and research expertise to grow the Indiana CTSI podcast participation and increase audience engagement.

- We will eventually allow the nation-wide network of CTSAs to pitch guests and shows covering Translational Research, and invite local CTSA leadership or faculty to participate in the podcast.

\section{RESULTS/ANTICIPATED RESULTS:}

- Utilizing quantitative analytics, we expect to see a significant increase in podcast downloads and listeners as we expand our offering to other CTSAs, beyond Indiana

- We expect that the CTSA-wide podcast will increase the nationwide level of knowledge and understanding of NCATS, translational research, and its benefits to society and healthcare.

- We anticipate, through this expanded podcast offering, a growing number of community members who are informed and engaged on the topics of translational research, clinical and translational sciences and beyond.

\section{DISCUSSION/SIGNIFICANCE OF IMPACT:}

- Podcasts are a convenient, portable, and efficient form of science communication.

- Podcasts also make information personal and offer us an excellent and innovative communications vehicle to spread the word about translational research, as well as the excellent work happening at each of our CTSAs.

4475

Meeting Partners Where They Are: Tailoring CommunityEngaged Research Consultation Services

Adam Paberzs ${ }^{1}$, Patricia Piechowski², Jordan Poll ${ }^{2}$, Meghan

Spiroff ${ }^{2}$, Karen Calhoun ${ }^{2}$, Ayse Buyuktur ${ }^{2}$, Athena McKay ${ }^{2}$, Donald Vereen $^{3}$, and Susan Woolford ${ }^{4}$

${ }^{1}$ University of Michigan School of Medicine; ${ }^{2}$ Michigan Institute for Clinical and Health Research; ${ }^{3}$ Community-Based Public Health, University of Michigan; ${ }^{4}$ University of Michigan, Pediatrics

OBJECTIVES/GOALS: One of the most significant challenges to community engagement experienced by Clinical and Translational Science Award (CTSA) institutions is inadequate capacity of academic and community partners to engage in collaborative research. Several CTSAs within the consortium provide consultation services to help address this gap. METHODS/STUDY POPULATION: For over 10 years, the Michigan Institute for Clinical and Health Research (MICHR), a CTSA at the University of Michigan, has provided CEnR-specific consultations to partners seeking support for a variety of needs. Consultations can be requested for assistance with identifying potential partners, developing partnership infrastructure, finding CEnR funding opportunities, and incorporating CEnR 